\title{
A High Visual Quality Embedding Method in Edges Based on Pixel Pair Difference
}

\author{
Yuan-bo Ma* \\ Dept. of Elec. Comm. and Sw. Eng. \\ Nanfang College of Sun Yat-Sen \\ University, GZ, China \\ mayb1314@foxmail.com \\ Ze-ming Lin \\ Dept. of Elec. Comm. and Sw. Eng. \\ Nanfang College of Sun Yat-Sen \\ University, GZ, China \\ Lin.zeming14@gmail.com
}

\author{
Quan-si Wen \\ Dept. of Elec. Comm. and Sw. Eng. \\ Nanfang College of Sun Yat-Sen \\ University, GZ, China \\ qwen2012@foxmail.com \\ Tung-shou Chen \\ Dept. of Computer Sci. and Inf. Eng. \\ National Taichung University of \\ Science and Technology, Taiwan \\ ttschen@gmail.com
}

\author{
Wien Hong \\ Dept. of Elec. Comm. and Sw. Eng. \\ Nanfang College of Sun Yat-Sen \\ University, GZ, China \\ wienhong@foxmail.com
}

\begin{abstract}
In this paper, we proposed a new data hiding method based on diamond encoding (DE) and pixel pair difference (PPD). DE proposes a pixel-wise algorithm which flexibly embeds different base digits to maximize payload and image visual quality. During DE embedding, digits embed in sequence without separately consider smooth areas and complex areas. We propose a method based on human visual system in that human eyes are more sensitive to the change of smooth areas where pixel pairs possess less difference. Embedding secret message in complex areas insignificantly affects visual quality of the image, correspondingly makes the stego image less detectable. The proposed method uses PPD to adaptively embed secret message in complex areas and thus improves DE performance. Experimental results illustrate that our algorithm has the same distortion with $\mathrm{DE}$ but provides a better visual quality of stego image.
\end{abstract}

\section{Categories and Subject Descriptors} I.4.9 [Image Process and Computer Vision] : Applications

\section{General Terms}

Algorithms, Experimentation, Security.

\section{Keywords}

Data hiding, Diamond encoding, Pixel pair difference, Human visual system, Switch division algorithm.

\section{INTRODUCTION}

Data hiding is embedding secret data (text, image, etc.) into digital media to transmit data secretly. The digital media, referred to various types of media, is used to "cover" the secret. After embedding, the cover that contains information is called "stego". To make secret information as undetectable as possible, the difference between cover and stego should be minimized.

The LSB method is a well-known data hiding method. This method simply replaces the least significant bits (LSB) of cover pixel with secret data via a small amount of calculation. Inspired by essential LSB philosophy, many advanced schemes are derived, such as Optimal Pixel Adjustment Process (OPAP) [1] and LSB Matching (LSBM). Each data hiding scheme contributes a further development since the image distortion is greatly reduced. One remarkable improvement is pair-wise embedding technique [3]. This technique exploits a pair of pixels as a unit for embedding a digit in a specific base. LSB Matching Revisited (LSBMR) [7], Exploiting Modification Direction (EMD) [11] and Diamond Encoding (DE) [2] are representative schemes. In these pair-wise embedding techniques, the produced visual quality of stego images is still limited since the same amount of data bits are embedded into pixels. Recently some methods embed different amounts of data bits into pixels based on human visual system (HVS). Pixel Value Difference (PVD) [9] and its variant, MFPVD [8], EA-PVD [10], APPM [4] and DE-MBNS [5] are of these types which adaptively embed secret digits in multiple bases which minimize the distortion while maximum the visual quality.

Among the aforementioned methods, DE and PVD scheme are the foundation to increase embedding efficiency because of pair-wise and multiple-base philosophy. This paper exploits the essential idea of PVD and proposes a new method based on DE and PPD. The distortion caused by embedding in the proposed method is less detectable compared with that of the DE method. In the rest of this paper, we first briefly introduce DE method in Section 2. Section 3 is the demonstration of proposed method. We suggest a switch division algorithm (SDA) to find the stego pixel pairs with minimize difference. All the experimental results are presented in Section 4. The end is conclusion section.

\section{DIAMOND ENCODING}

DE method embeds one digit $S$ into two pixels by using $l$-ary notation system. The parameter $l$ is calculated by

$$
l=2 k^{2}+2 k+1 \text {, }
$$

where $k$ is the embedding parameter. Firstly, the original images generate non-overlapping diamond blocks of two consecutive pixels $(a, b)$. Each diamond characteristic value (DCV) is computed by the following equation:

$$
f(a, b)=\bmod ((2 k+1) a+b, l),
$$

where $f(a, b)$ also defined as extraction function. Suppose each diamond block consists of a neighborhood set $S_{k}\left(p_{i, 1}, p_{i, 2}\right)$. This set represents all the pixel vectors $\left(p_{i, 1}, p_{i, 2}\right)$ with the distance to vector $(a, b)$ smaller than $k$. The vectors $\left(p_{i, 1}, p_{i, 2}\right)$ can be calculated by

$$
S_{k}(p, q)=\left\{(a-b)|| p_{i, 1}-a|+| p_{i, 2}-b \mid \leq k\right\} .
$$


Secondly, the DE method searches the DCV in set $S_{k}\left(p_{i, 1}, p_{i, 2}\right)$ to find a vector $\left(p_{i, 1}^{\prime}, p_{i, 2}^{\prime}\right)$ which the DCV is equal to $S$. Then replace the cover pixel $(a, b)$ by $\left(p_{i, 1}^{\prime}, p_{i, 2}^{\prime}\right)$. The difference between cover pixel and stego pixel is never larger than $k$ by applying DE scheme which has been determined by the diamond search block.

Secret digit can be simply extracted by employing the extraction function with stego pixel pair $\left(p_{i, 1}^{\prime}, p_{i, 2}^{\prime}\right)$. For clearly explanation of $\mathrm{DE}$, here we use a figure to illustrate diamond block in different $k$.

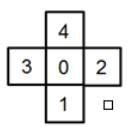

$k=1$, Base $=5$

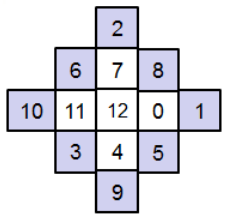

$k=2$, Base $=13$

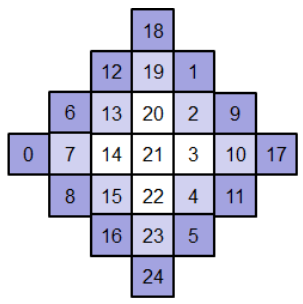

$k=3$, Base $=25$
Figure 1. Diamond search block with $k=1, k=2, k=3$

Here is a simple example for the DE method. Suppose $k=2$ and use eq. (1) to get the base $l=13$. Assume the secret digits $1_{13}$ and $5_{13}$ will be embedded into cover pixel pairs $(3,12)$ and $(4,7)$, respectively. DCV of $(3,12)$, which can be computed from eq. (2), is 1 . Since the DCV is equal to secret digit $1_{13}$, the stego pixel pair is the same as cover pixel pair. Likewise, for the second secret digit, the DCV of $(4,7)$ is 3 . Since the DCV is not equal to secret digit $5_{13}$, replacing the pixel pair $(4,7)$ by $(6,7)$ of which the DCV is $5_{13}$. After diamond encoding, the stego pixel are $(3,12)$ and $(6,7)$. The procedure is illustrated in figure 2 . The embedded digits can be extracted from stego pixel pair $(3,12)$ and $(6,7)$ by using the extraction function eq. (2).

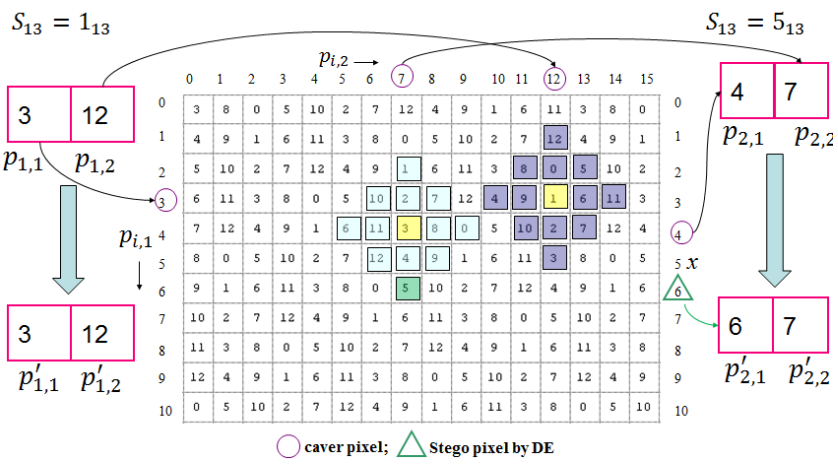

Figure 2. Illustration of Diamond encoding

\section{PROPOSED METHOD}

In this section, we present a pixel pair difference (PPD) based diamond encoding scheme. Compared with other pair-wise methods, DE method extends the payload by embedding digits in a larger notational system. Both LSBMR scheme and EMD scheme suffer from an insufficient payload problem since payload has been limited by the embedding base. In LSBMR, one pixel pair embeds 2 bits at once. So the averaged payload is $1 \mathrm{bpp}$. Meanwhile, EMD employs $n$ pixels as an embedding unit and embeds digits in $(2 n+1)$ base. The maximum payload occurs at $n=2$ and the payload is $(1 / 2) \log _{2} 5 \approx 1.16 \mathrm{bpp}$. Inspired by EMD, DE method greatly improves payload by using a parameter $k$ to determine the secret digit base $l$. Suppose $k=2$, then the digit base $l$ is 13 , correspondingly the payload increases to $(1 / 2) \log _{2} 13 \approx 1.85$ bpp.

In conclusion, $\mathrm{DE}$ offers extendable payload with acceptable image quality. But once we consider HVS, DE algorithm still has improvement. During DE, $l$-ary secret digits are embedded into cover pixel pairs successively without distinguishing smooth areas and complex areas. For a HVS based data hiding scheme, different amount of data are embedded into smooth and complex areas to make the stego image more undetectable while maximizing the payload. The proposed method skips these smooth areas and embeds data only in complex areas. As a result, the pixels with large distortion concentrate in complex areas where human eyes tolerate more change. The proposed method not only improves the visual quality of stego image, but also affects the payload and efficiency as less as possible.

\subsection{Threshold Determination}

The proposed method uses the absolute difference $d_{i}=$ $\left|p_{i, 1}-p_{i, 2}\right|$ of pixel pairs, which we named as PPD, to distinguish the smooth areas and complex areas. To minimize the distortion, pixel value in range $[0,255]$ is divided into two divisions by a threshold parameter $T$ in figure 3 . Pixel pairs with difference smaller than $T$ are classified into the un-embeddable division, where no secret digit is embedded. Therefore the stego pixels are the same as the cover pixels without any distortion. Contrary to the un-embeddable division, pixel pairs with difference more than $T$ are divide into the embeddable division, in which efficiently embeds the secret digits by DE scheme.

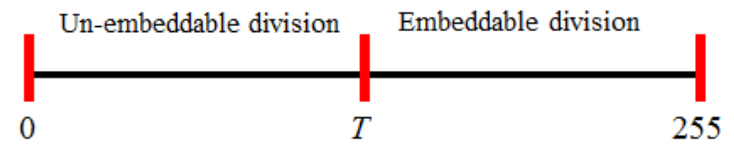

Figure 3. Division classification

Since the proposed method embeds secret digits in the pixel pairs with difference larger than a threshold $T$, our proposed method requires determining a largest $T$ such that all the secret digits can just be embedded.

Let $N_{d}(T)$ be the number of pixel pairs $\left(p_{i, 1}, p_{i, 2}\right)$ satisfying $d_{i}=$ $\left|p_{i, 1}-p_{i, 2}\right|>T$. To embed $|S|$ secret digits $S$, where $|S|$ is the length of $S$, we simply find the largest $T$ satisfying $N_{d}(T) \geq|S|$. The threshold $T$ can be easily solved by calling bisection method or other equation solving method.

\subsection{Switch Division Algorithm}

As we demonstrated before, extraction only processes with stego pixel pairs sit in embeddable division. However under a certain condition, stego pixel pair should switch into un-embeddable division after DE scheme. For example, in figure $2,\left(p_{2,1}, p_{2,2}\right)=$ $(4,7), d_{2}=3$, secret is $5_{13}$. After diamond encoding, $\left(p_{2,1}^{\prime}, p_{2,2}^{\prime}\right)=$ $(6,7), d_{2}^{\prime}=1$. If $T$ is 2 or 3 , this stego pixel pair has fallen into unembeddable division due to $d_{i}^{\prime}$ less than $T$. In this case, the secret digit $5_{13}$ should be lost. We mark this kind of cover pixel pair as the to-be-destroyed pixel pair which should not embed data at all. We propose a switch division algorithm (SDA) to exchange the to-be-destroyed cover pixel pair into the un-embeddable division, while keeping the distortion minimal.

Let the to-be-destroyed pixel pair be $\left(p_{i, 1}, p_{i, 2}\right)$ and $d=$ $\left|p_{i, 1}-p_{i, 2}\right|>T$, we desire to replace $\left(p_{i, 1}, p_{i, 2}\right)$ by $\left(p_{i, 1}^{\prime \prime}, p_{i, 2}^{\prime \prime}\right)$ at 
the lowest distortion while satisfying $\left|p_{i, 1}^{\prime \prime}-p_{i, 2}^{\prime \prime}\right|<T$. The best $\left(p_{i, 1}^{\prime \prime}, p_{i, 2}^{\prime \prime}\right)$ can be obtained by solving the optimization problem:

$\operatorname{Minimize}\left(p_{i, 1}-p_{i, 1}^{\prime \prime}\right)^{2}+\left(p_{i, 2}-p_{i, 2}^{\prime \prime}\right)^{2}$

Subject to: $\left|p_{i, 1}^{\prime \prime}-p_{i, 2}^{\prime \prime}\right|<T$

Once SDA scheme implemented, it is not hard to conclude the $\left(p_{i, 1}^{\prime \prime}, p_{i, 2}^{\prime \prime}\right)$ always located around the middle of $p_{i, 1}^{\prime \prime}$ and $p_{i, 2}^{\prime \prime}$.

\subsection{Embedding Procedure}

Let $I$ be the cover image of size $M_{1} \times M_{2}$, and $S$ be the secret digits of length $|S|$. The embedding procedures are listed as following:

Step 1. Partition the cover image into non-overlapping pixel pair $I=\left\{\left(p_{i, 1}, p_{i, 2}\right), 1 \leq i \leq N\right\}$, where $N$ is the total number of pixel pairs. Calculate the threshold $T$ to define division to which each pixel pair belongs, as described in Section 3.1.

Step 2. Process the embedding algorithm. For each pixel pair $\left(p_{i, 1}, p_{i, 2}\right)$, if $d_{i} \leq T$, the pixel pair is classified in the unembeddable division. As a result, we skip embedding and set the stego pixel pair $\left(p_{i, 1}^{\prime}, p_{i, 2}^{\prime}\right)=\left(p_{i, 1}, p_{i, 2}\right)$; if $d_{i}>T$, the pixel pair is in embeddable division, embedding one secret digit by DE method and obtain a stego pixel pair $\left(p_{i, 1}^{\prime}, p_{i, 2}^{\prime}\right)$. Else $d_{i}^{\prime}=\left|p_{i, 1}^{\prime}-p_{i, 2}^{\prime}\right| \leq T$, switch $\left(p_{i, 1}^{\prime}, p_{i, 2}^{\prime}\right)$ to un-embeddable division using the proposed SDA.

Step 3. Take the next cover pixel pair and repeat step 2 until all $|S|$ secret digits are embedded.

\subsection{Extraction Procedure}

Once obtain the stego image $I^{\prime}$, threshold $T,|S|$, the embedded digits $S$ can be extracted by eq. (2). The detail steps are listed as following:

Step 1. Partition the stego image into non-overlapping pixel pair $\left(p_{i, 1}^{\prime}, p_{i, 2}^{\prime}\right)$, where $1 \leq i \leq N$.

Step 2. Use the same scanning order as in the embedding procedure to obtain the difference $d_{i}^{\prime}=\left|p_{i, 1}^{\prime}-p_{i, 2}^{\prime}\right|$. If $d_{i}^{\prime} \leq T$, the pixel pair is in un-embeddable division, and no secret is embedded, move to next stego pixel pair; if $d_{i}^{\prime}>T$, the pixel pair is in embeddable division, extracting one secret digit using eq. (2), $S=f\left(p_{i, 1}^{\prime}, p_{i, 2}^{\prime}\right)$.

Step 3. Take the next stego pixel pair and repeat step 2 until all $|S|$ secret digits are extracted.

\subsection{Simple Example}

The aforementioned example is referred to explain our procedure. Let cover pixel pairs be $(3,12)$ and $(4,7)$. Secret digits are $1_{13}$ and $5_{13}$. Supposed $k=2, T=2$, as showed in figure 4 .

Since $d_{1}=|3-12|=9>T,(3,12)$ sits in embeddable division. We have DCV $=f(3,12)=1_{13}$, thus stego pixel pair is the same as covel pixel. As a result, stego pixle pair $(3,12)$ is still located in embeddable division. For the pixel pair $(4,7), d_{2}=$ $|4-7|=3>T,(4,7)$ sits in embeddable division. DCV = $f(4,7)=3 \neq 5_{13}$. Use DE scheme get $\operatorname{DE}(4,7)=(6,7)$. After doging this, stego pixle pair $(6,7)$ switches into un-embeddable division since $d_{2}^{\prime} \leq T$. Therefore we recalculate stego pixel pair use SDA and obtain $\operatorname{SDA}(4,7)=(5,6)$. By only applying DE method, the the difference bwteen cover pixel pair $(4,7)$ and stego pixle pair $(6,7)$ is 4 . But by applying our propsed algorithm, the difference between cover pixel pair $(4,7)$ and stego pixle pair $(5,6)$ is only 2 .

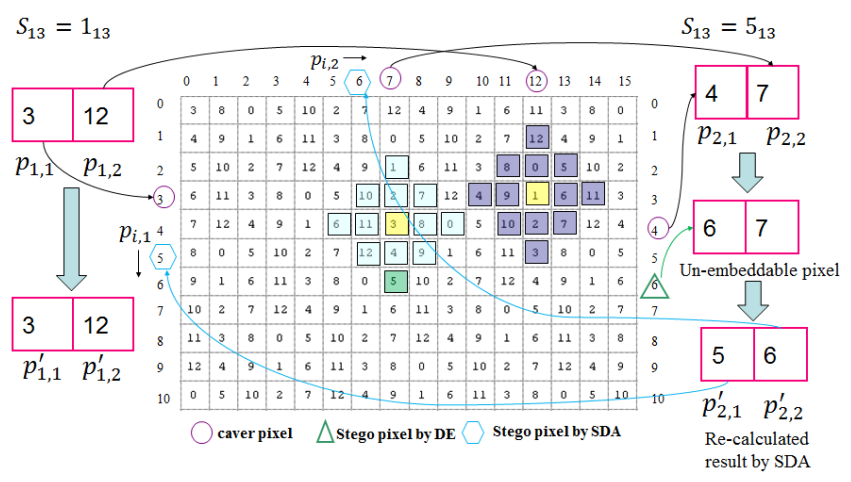

Figure 4. Illustration of proposed method

\section{EXPERIMENTAL RESULTS}

In this section, several experiments are performed to analyze proposed method and compare with the Diamond Encoding method in terms of image quality, PSNR and SSIM [6]. SSIM is employed to measure the similarity of two images. The value of SSIM is within the range $[-1,1]$. The more SSIM value close to 1 , the more two images are similar to each other. Three standard gray images will be referred as cover images, including Lena, Lighthouse and Baboon, as shown in figure 5. All images are $512 \times 512$ pixels and were obtained from SIPI image databases and internet, respectively.

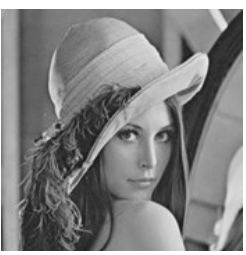

(a) Lena

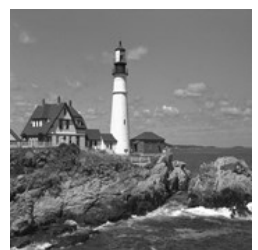

(b) Lighthouse

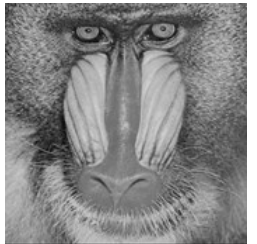

(c) Baboon
Figure 5. Three standard images

In figure 6, we show the comparison result of the difference of cover images and stego images by applying proposed method in the order of Lena, Lighthouse and Baboon.

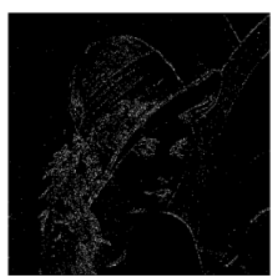

(a) $T=13$

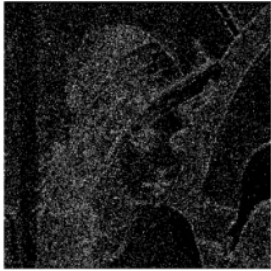

(d) $T=4$

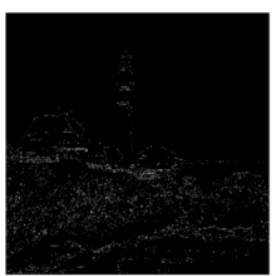

(b) $T=57$

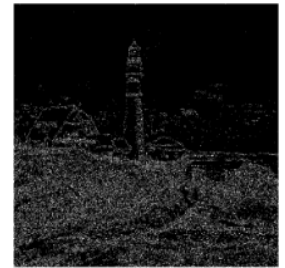

(e) $T=10$

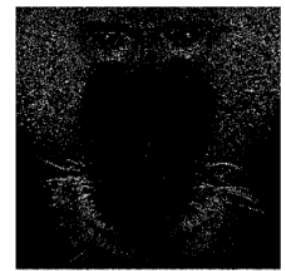

(c) $T=41$

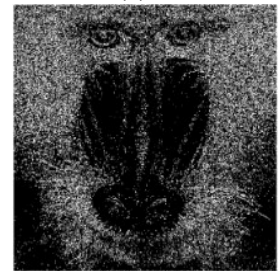

(f) $T=13$
Figure 6. Diff. of cover images and stego images 
(a) $\sim$ (c) are 10,000 digits payload and (e) (f) are 50,000 digits payload. To obtain these secret digits, we randomly create a secret sequence to embed. We use $k=2$ in the experiments. DE scheme embeds secret in sequence while proposed scheme embeds adjustably due to PPD. The white spots mean embedded position while black spots do not embed secret, and the embedding location shows that the complexity of cover image influences the embedding location.

Table 1. Comparison result of PSNR and $T$.

\begin{tabular}{|c|c|c|c|c|}
\hline \multirow{2}{*}{ payload } & \multicolumn{2}{|c|}{$\begin{array}{c}\text { Payload=10,000 } \\
\text { (digits) }\end{array}$} & \multicolumn{2}{c|}{$\begin{array}{c}\text { Payload=50,000 } \\
\text { (digits) }\end{array}$} \\
\cline { 2 - 5 } & PSNR(dB) & $\boldsymbol{T}$ & PSNR(dB) & $\boldsymbol{T}$ \\
\hline Lena & 59.07 & 13 & 52.36 & 4 \\
\hline Lighthouse & 60.70 & 41 & 53.67 & 10 \\
\hline Baboon & 58.94 & 57 & 52.10 & 17 \\
\hline
\end{tabular}

Table 1 summarizes the PSNR and $T$ of three standard images with various payloads by using proposed method. First, we compare the same cover image with different payload. As expected, the growth of $T$ dose depends on the value of payload. At the same time, the white points increase. Second, we compare the same payload with different cover images. The more complexity of cover image, the larger $T$ generates.

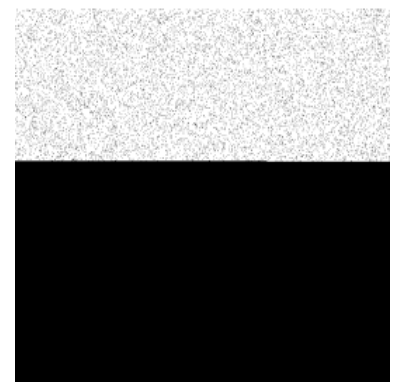

(a) Diff. of DE Scheme

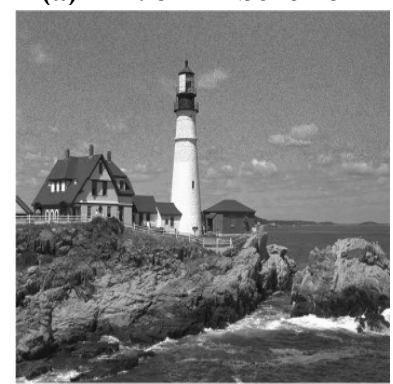

(c) Stego of DE Scheme SSIM $=0.86$

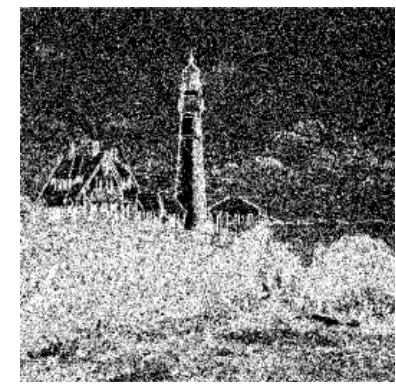

(b) Diff. of Proposed Method

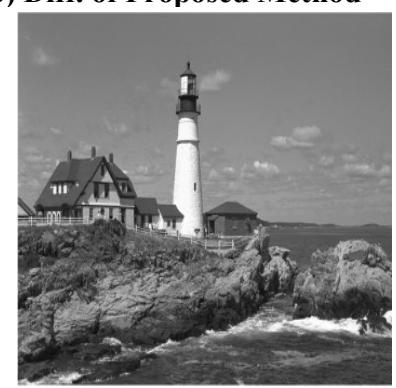

(d) Stego of Proposed Method SSIM $=0.98$
Figure 7. Comparison result of Lighthouse.

Figure 7 indicates the comparison result of Lighthouse produced by DE method and proposed scheme. (a) and (b) are differences of cover images and stego images. (c) and (d) are stego images. Let $k=30$, we embed the same payload with 80,000 secret digits into Lighthouse. The difference between the cover image and stego image indicates that the DE scheme only embed the upper part of cover image in turn. On the other hand, our proposed method adjustably embeds secret digits into the complex pixel pairs. The difference of stego image between DE scheme and proposed scheme is visually different. Meanwhile, the SSIM of DE scheme is smaller than the SSIM of proposed scheme, which means proposed method improves the visual quality greatly.

\section{CONCLUSION}

This paper proposed a data embedding method with the consideration of human visual system based on DE and PPD scheme. Two pixels are scanned as an embedding unit and conceal digits adaptively according to the absolute difference of pixel pairs. The contributions of the proposed method are summarized as follows. Firstly, our proposed method embeds data in all pixel pairs distinctively among smooth areas and complex areas which improve the image visual quality. Secondly, our proposed method raises a SDA to detect the to-be-destroyed pixel pairs then switch them to un-embeddable division with minimal distortion. The experimental results reveal the improvement which could be discovered visually.

\section{REFERENCES}

[1] Chan, K. and Cheng, L.M. 2004. Hiding Data in Images by Simple LSB Substitution. Pattern Recognition. vol. 37, no. 3, 469-474.

[2] Chao, R.M., Wu, H.C., Lee, C.C., and Chu, Y.P. 2009. A Novel Image Data Hiding Scheme with Diamond Encoding. EURASIP Journal on Information Security. vol. 2009, Article ID 658047.

[3] Hong, W. 2013. Adaptive Image Data Hiding in Edge Using Patched Reference Table and Pair-Wise Embedding Technique. Information Science. vol. 221, 473-489. DOI= http://dx.doi.org/10.1016/j.ins.2012.09.013.

[4] Hong, W. and Chen, T.S. 2012. A Novel Data Embedding Method Using Adaptive Pixel Pair Matching. IEEE Transactions on Information Forensics and Security. vol. 7, no. 1. DOI= http://dx.doi.org/10.1109/TIFS.2011.2155062

[5] Hong, W., Chen, T.S., and Luo, C.W. 2012. Data Embedding Using Pixel Value Differencing and Diamond Encoding with Multiple-base Notational System. Journal of System and Software. vol. 85, iss, 5, 1166-1175. DOI= http://dx.doi.org/10.1016/j.jss.2011.12.045

[6] Hong, W., Chen, T.S., and Wu M.C. 2013. An Improved Human Visual System Based Reversible Data Hiding Method Using Adaptive Histogram Modification. Information Science. vol. 291, 87-97. DOI= http://dx.doi.org/10.1016/j.optcom.2012.10.081.

[7] Mielikainen, J. 2006. LSB Matching Revisited. IEEE Signal Processing Letters, vol. 13, no. 5, 285-287.

[8] Wang, C.M., Wu, N.I., Tsai, C.S., and Hwang, M.S. 2008. A High Quality Steganographic Method with Pixel-Value Differencing and Modulus Function. Journal of Systems and Software. vol. 81, no. 1, 150-158. DOI= http://dx.doi.org/10.1016/j.jss.2007.01.049

[9] Wu, D.C., and Tsai, W.H. 2003. A Steganographic Method for Images by Pixel-Value Differencing. Pattern Recognition Letters. vol. 24, 1613-1626. DOI= http://dx.doi.org/10.1016/S0167-8655(02)00402-6

[10] Yang, C.H., Weng, C.Y., Wang, S.J., and Sun, H.M. 2008. Adaptive Data Hiding in Edge Areas of Images with Spatial LSB Domain Systems. IEEE Transactions on Information Forensics and Security. vol. 3, no. 3, 488-497. DOI= http://dx.doi.org/10.1109/TIFS.2008.926097

[11] Zhang, X. and Wang, S. 2006. Efficient Steganographic Embedding by Exploiting Modification Direction. IEEE Communications Letters. vol. 10, no. 11, 781-783. DOI=http://dx.doi.org/10.1109/LCOMM.2006.060863 